\title{
The Emerging Role of Exosomes in Oral Squamous Cell Carcinoma
}

\author{
Yanhui Lu ${ }^{1,2+}$, Zhichao Zheng ${ }^{1,2 t}$, Yunyi Yuan ${ }^{3 t}$, Janak L. Pathak ${ }^{1}$, Xuechao Yang ${ }^{1,2}$, \\ Lijing Wang ${ }^{1,4}$, Zhitong Ye ${ }^{1}$, William C. Cho ${ }^{5 *}$, Mingtao Zeng ${ }^{* *}$ and Lihong Wu ${ }^{1,2 *}$
}

\section{OPEN ACCESS}

Edited by:

Eduardo Marbán

Independent Researcher, Los Angeles, United States

Reviewed by: Jordan Cheng,

University of California, Los Angeles,

United States

Hamed Mirzaei,

Kashan University of Medical

Sciences, Iran

Jafar Rezaie,

Urmia University of Medical Sciences,

Iran

*Correspondence: Lihong Wu

wcanhong@163.com Mingtao Zeng

Mt.Zeng@ttuhsc.edu

William C. Cho

chocs@ha.org.hk

${ }^{\dagger}$ These authors have contributed equally to this work and share first

authorship

Specialty section:

This article was submitted to

Molecular Medicine,

a section of the journal

Frontiers in Cell and Developmental

Biology

Received: 11 November 2020

Accepted: 03 February 2021

Published: 22 February 2021

Citation:

Lu Y, Zheng Z, Yuan Y, Pathak JL,

Yang $X$, Wang $L$, Ye Z, Cho WC,

Zeng $M$ and Wu L (2021) The

Emerging Role of Exosomes in Oral

Squamous Cell Carcinoma.

Front. Cell Dev. Biol. 9:628103.

doi: 10.3389/fcell.2021.628103
${ }^{\prime}$ Affiliated Stomatology Hospital of Guangzhou Medical University, Guangzhou Key Laboratory of Basic and Applied Research of Oral Regenerative Medicine, Guangzhou, China, ${ }^{2}$ Guangzhou Medical University School and Hospital of Stomatology, Guangzhou, China, ${ }^{3}$ Department of Orthodontics, Affiliated Stomatology Hospital of Guangzhou Medical University, Guangzhou Key Laboratory of Basic and Applied Research of Oral Regenerative Medicine, Guangzhou, China, ${ }^{4}$ Vascular Biology Research Institute, Guangzhou Higher Education Mega Center, Guangdong Pharmaceutical University, Guangzhou, China, ${ }^{5}$ Department of Clinical Oncology, Queen Elizabeth Hospital, Kowloon, Hong Kong, ${ }^{6}$ Center of Emphasis in Infectious Diseases, Department of Molecular and Translational Medicine, Paul L. Foster School of Medicine, Texas Tech University Health Sciences Center El Paso, EI Paso, TX, United States

Oral cancer constitutes approximately $2 \%$ of all cancers, while the most common type, oral squamous cell carcinoma (OSCC) represents $90 \%$ of oral cancers. Although the treatment of OSCC has improved recently, it still has a high rate of local recurrence and poor prognosis, with a 5-year survival rate of only 50\%. Advanced stage OSCC tends to metastasize to lymph nodes. Thus, exploring new therapeutic strategies for OSCC is therefore an urgent priority. Exosomes, the small membrane vesicles derived from endosomes, have been detected in a wide array of bodily fluids. Exosomes contain a diversity of proteins, mRNAs, and non-coding RNAs, including microRNAs, long non-coding RNAs, piRNAs, circular RNAs, tsRNAs, and ribosomal RNAs, which are delivered to neighboring cells or even transported to distant sites. Exosomes have been associated with the tumorigenesis of OSCC, promote the proliferation, colonization, and metastasis of OSCC by transferring their contents to the target cells. Furthermore, exosomes are involved in the regulation of the tumor microenvironment to transform conditions favoring cancer progression in vivo. In this review, we summarize the crucial role of exosomes in the tumorigenesis and progression of OSCC and discuss the potential clinical application of exosomes in OSCC treatment.

Keywords: oral squamous cell carcinoma, non-coding RNAs, exosomes, tumor microenvironment, extracellular vesicles

\footnotetext{
Abbreviations: OSCC, oral squamous cell carcinoma; EVs, extracellular vesicles; MVB, multivesicular body; CHB, chronic hepatitis B; TME, tumor microenvironment; ESCRT, endosomal tethering complexes necessitated for transport; GTPase, Rab guanosine triphosphatase; PEG, Polyethylene glycol; ncRNAs, non-coding RNAs; miRNAs, microRNAs; hnRNPA2B1, heterogeneous nuclear ribonucleoprotein A2B1; CAFs, cancer-associated fibroblasts; PTEN, phosphatase and tensin homolog; PDCD4, programmed cell death 4; Mark1, microtubule-affinity-regulating kinase 1; TGFBR1, type I TGFbeta receptor; SOCS1, suppressor of cytokine signaling 1; TPM1, tropomyosin alpha-1 chain; CHD9, chromodomain helicase DNA binding protein 9; WRN, Werner syndrome gene; DENND2D, DENN/MADD domain containing 2D; ECM, extracellular matrix; EMT, epithelial-mesenchymal transition; MMPs, extracellular matrix proteins; NFs, normal fibroblasts; BCL2, B-cell lymphoma 2; Tregs, regulatory T cells; MDSCs, myeloid-derived suppressor cells; TEX, hypoxic tumorderived exosome; lncRNAs, long non-coding RNAs; circRNAs, circular RNAs; piRNAs, P-element-induced wimpy testis (PIWI)-interacting RNAs; rRNAs, ribosomal RNAs; tsRNAs, transfer RNAs; HMGA1, the high mobility group A1; USP7, ubiquitin-specific protease-7; ARHGAP11A, Rho GTPase activating protein 11A; ATMs, ataxia telangiectasia mutated; AMPK, adenosine monophosphate-activated protein kinase; TRAP1, Tumor necrosis factor receptor-associated protein 1; EGFR, epidermal growth factor receptor; HSP, heat shock protein; PF4V1, platelet factor 4 variant 1; CXCL7, C-X-C motif chemokine ligand 7; F13A1, Coagulation factor XIII A chain; ApoA1, apolipoprotein A1; VEGF, vascular endothelial growth factor; LNM, lymph node metastasis; ROC, receiver operating characteristic.
} 


\section{INTRODUCTION}

Oral squamous cell carcinoma is the most common subtype of oral carcinoma, a genetic and epigenetic disease characterized by histopathological differentiation and a propensity for LNM (Bu et al., 2015). Surgical removal of tumors as well as pre- or postoperative chemotherapy, radiotherapy, and adjuvant therapies are the main strategies to increase survival (Coyte et al., 2014; Adel et al., 2016). Although treatment outcomes for OSCC have been improved recently, the prognosis for OSCC is still poor, the 5 -year survival rate is reported as $50 \%$, due to late diagnosis which resulting infeasibility of curative resections. Furthermore, the prognosis for this disease is poor due to metastatic invasion, with a propensity for local recurrence and distant metastasis (Huang et al., 2019). OSCC dissemination targets both local tissues via direct invasion and distant sites by seeding pre-metastatic niches through secreted elements, including exosomes (Qiu et al., 2019). Considering the risk of late diagnosis of OSCC, improvements in prevention, early diagnosis, and treatment efficacy are urgently needed (Csõsz et al., 2017).

According to the International Society for Extracellular Vesicles (ISEV), EVs are nano-size lipid bilayer vesicles released naturally from the cells to the ECM (Kalra et al., 2012; Thery et al., 2018; Jabbari et al., 2020b) (Figure 1). Generally, EVs are categorized as exosomes, microvesicles, and apoptotic bodies (Ahmadi and Rezaie, 2020; Jabbari et al., 2020a). Exosomes are small, membranous, extracellular microvesicles $(\sim 30-150 \mathrm{~nm}$ in diameter) of endocytic origin. The formation of exosomes includes the beginning, endocytosis, MVB creation, and finally exosome secretion (Mellman, 1996; Keller et al., 2006; Raposo and Stoorvogel, 2013; Abak et al., 2018). Previous studies showed that non-coding RNAs (ncRNAs), mRNAs, proteins, and DNA fragments can be carried as "cargo" in EVs, which could serve as novel diagnostic biomarkers for OSCC (Raposo and Stoorvogel, 2013; Abak et al., 2018; Gai et al., 2018; Pourhanifeh et al., 2020). Another study found that exosomes can reprogram signal transduction under pathophysiological conditions and deliver important proteins as mediators ( $\mathrm{Li}$ et al., 2019d; Amiri et al., 2021). These studies demonstrated that exosomes are present in the saliva of healthy donors, and exosomes have been reported in other body fluids, such as blood, cerebrospinal fluid, serous cavity effusion, and urine (Cao et al., 2020). Exosomes play an essential role in mediating signal transport for intercellular communication or over long distances by transporting microRNA (miRNAs), mRNAs, and proteins (Ohno et al., 2013a; Zhang et al., 2015; Sadri Nahand et al., 2020). Exosomal miRNAs are potential diagnostic biomarkers for various malignancies, regulating protein expression in cell proliferation, tumor metastasis, cell apoptosis, genomic instability, and immune responses. Wang H. et al. (2014) showed that exosomal miR-21 in the serum of OSCC patients is higher than that in chronic hepatitis patients and healthy individuals, suggesting that serum miRNAs may act as diagnostic biomarkers for OSCC. Sohn et al. (2015) detected serum exosomal miRNAs in patients with $\mathrm{CHB}$, liver cirrhosis, and OSCC with $\mathrm{CHB}$ and discovered that a panel of eight exosomal miRNAs are significantly different between the OSCC and chronic hepatitis or liver cirrhosis groups. These efforts have revealed that exosomal contents can be transported across biological membranes and that exosomes have a role in OSCC development. Exosomes and their pattern of transfer deserve more attention to clearly define their roles in OSCC diagnosis and therapeutics. This review highlights exosomes in exchanges between normal and OSCC cells as well as the endocrine transport of exosomes from distant cells via the TME. We also review recent advances in exosomes in cancer initiation, progression and their potential clinical relevance to OSCC.

\section{EXOSOME BIOGENESIS, ISOLATION METHODS, AND BIOLOGICAL FUNCTIONS}

\section{The Biogenesis of Exosomes}

The intraluminal vesicles (ILVs) are formed through inside budding in the multivesicular bodies (MVB) during the maturation of early endosomes to late endosomes which is regulated by ceramide (Nahand et al., 2020a). The cargos are encapsulated into ILVs during budding. However, the mechanism controlling cargo sorting is rather complicated and still unclear (Leidal and Debnath, 2020). EVs have overlapping sizes, similar morphology, and unspecific contents, resulting in difficulties in the isolation of specific subpopulations (Mathieu et al., 2019). The ILVs fuse with the cell membrane and are released into the extracellular space (Nahand et al., 2020b). It has been demonstrated that endosomal-tethering complexes necessitated for transport (ESCRT)-dependent and independent activities play an essential role in MVB formation (Hashemian et al., 2020). The ESCRT members including ESCRT-0, -I, -II, -III, and the related AAA-ATPase Vps4 complex recognize ubiquitinated membrane proteins leading to their internalization within the multivesicular endosome (Asgarpour et al., 2020; Ghaemmaghami et al., 2020). The MVB trafficking and secretion of exosomes are regulated by several members including the GTPase family (Rab11, Rab27a, Rab27b, and Rab35), heparanase, soluble NSF attachment receptor, and cytoskeleton regulatory proteins (Pant et al., 2012; Azmi et al., 2013; Beach et al., 2014; Abak et al., 2018). Furthermore, the dissemination process of the exosomes requires cellular stress, such as oxidative stress, hypoxia, etc (Abak et al., 2018). The released exosomes could transport into the recipient cells by the interaction between receptor-ligand, membrane fusion, and endocytosis through phagocytosis (Abak et al., 2018).

\section{Exosome Isolation Methods}

The isolation methods of exosomes mainly include ultracentrifugation, size-based isolation, polymer precipitation, immunoaffinity, and microfluidic separation (Oeyen et al., 2018). Currently, ultracentrifugation is the most commonly used and the gold standard for exosome isolation. The advantage of ultracentrifuge is to treat lots of samples at one time. However, the problems of purity and damage the integrity of exosomes are the main disadvantages of the ultracentrifugation method (Tschuschke et al., 2020). PEG 


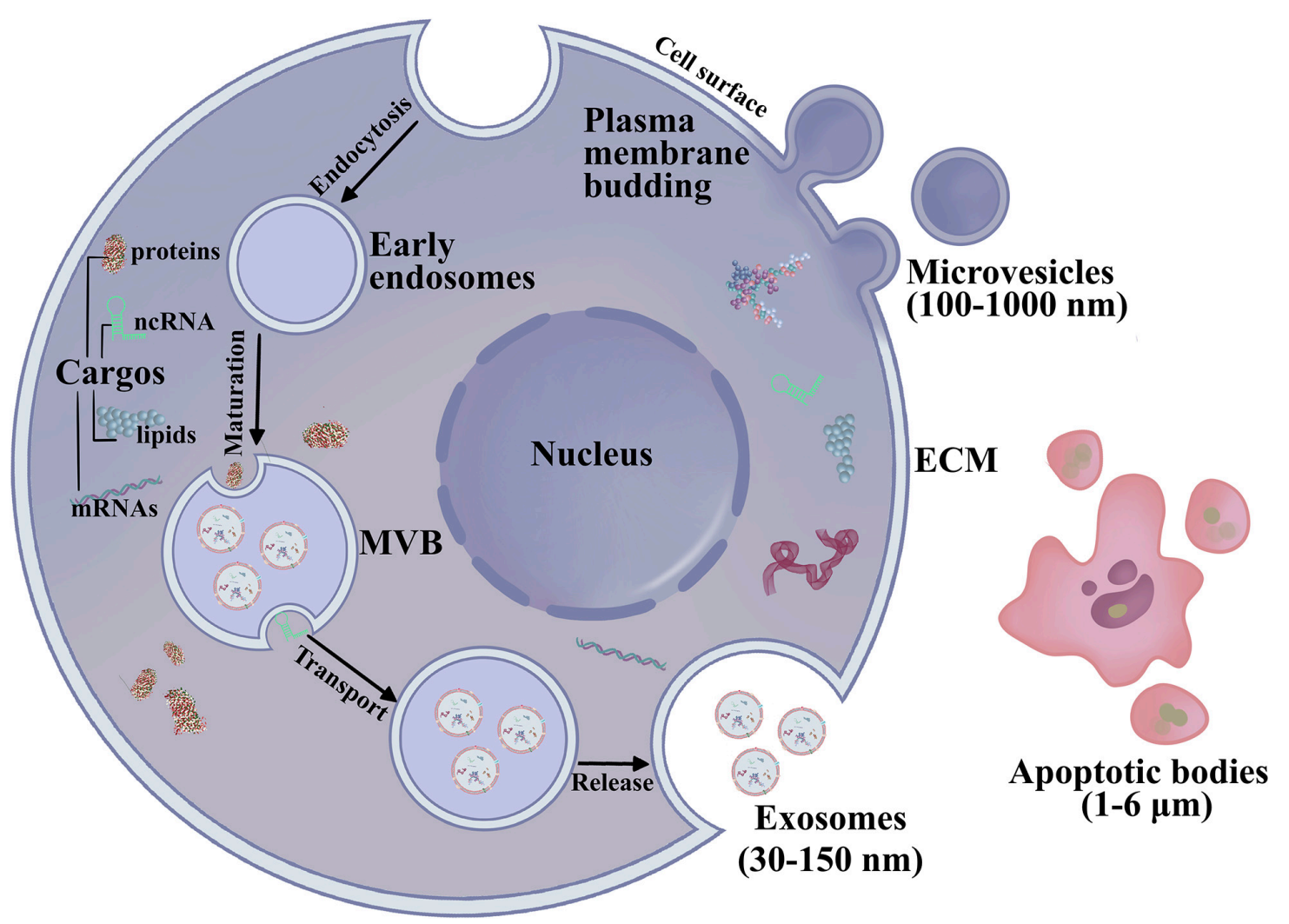

FIGURE 1 | The biogenesis and secretion of EVs. Exosomes (30-150 nm) are generated by membrane endocytosis. Microvesicles (100-1,000 nm) are formed by the plasma membrane budding. Microvesicles are irregular in shape and heterogeneous in population. The largest EVs are apoptotic bodies (1-6 $\mu \mathrm{m})$ are involved in the pathological condition.

polymer combines the water molecules of the exosomes and reduces the solubility. PEG-based exosome isolation can be done in low-speed centrifugation with high efficiency (Doyle and Wang, 2019). The failure of the selection of exosomes and other EVs is the main drawback of PEG precipitation. Size-based exosome isolation is based on ultrafiltration and size exclusion chromatography. Ultrafiltration membrane isolates the exosomes by cutoff molecular sizes, which may lead to the exosome damage due to the pressure ( $\mathrm{Li}$ et al., 2017). The size exclusion chromatography can be used to slow down the movement of small molecular, that protect the integrity and activity of exosomes. Magnetic bead and enzyme-linked immunosorbent assay are immunoaffinitybased exosome isolation methods, These methods capture the exosomes according to the antigen recognition of specific antibody (Zhang et al., 2020). Immunoaffinity-based exosome isolation generates pure exosomes with low yield. Microfluidic technology isolates exosomes in a short time with high purity, it differentiates physical and biochemical characteristics of exosomes by phosphatidylserine-specific proteins on exosomes (Jiang et al., 2020).

\section{The Biological Function of Exosomes}

Under physiological conditions, exosomes are important mediators of cell-cell and inter-tissue communication. Exosomes exhibit important functions in regulating cellular activities during physiological and pathological conditions. During the cancer progression, different cells such as cancer cells, immune cells in TME generate exosomes that can transfer nucleotides and proteins among cells and participate in the complex pathogenesis of tumor development and metastasis (Ridder et al., 2014; Nazimek et al., 2016). Increasing evidence indicates that tumor cells communicate both with other tumor cells and with normal cells present in the TME via secretion and transfer of exosomal contents. Exosomal contents regulate tumor growth, angiogenesis, metastasis, sensitivity to chemotherapy, and immune evasion. Thus, it is essential to explore the effects of exosomes on OSCC development in vitro and in vivo.

\section{EXOSOMAL MIRNAS ARE ESSENTIAL FOR OSCC DEVELOPMENT}

\section{miRNAs in Exosomes}

Among the bioactive components of exosomes, miRNAs can epigenetically alter gene function in the recipient cell, thus exerting their essential regulatory function on gene expression (Wang Y. et al., 2014). miRNAs are short ncRNAs of approximately 19-24 nucleotides in length (Chen et al., 2012b) and function to suppress the expression of protein-coding genes 
at the post-transcriptional level by degrading or inhibiting the translation of mRNAs (Bartel, 2004; Miska, 2005; Kim et al., 2009). Moreover, miRNAs and their target genes constitute complicated regulatory networks that contribute to the finetuning of various biological processes, such as cell proliferation, differentiation, and apoptosis (Miska, 2005; Hwang and Mendell, 2006). It has been demonstrated that miRNAs manipulate more than $30 \%$ of human genes, governing all cellular, physiological, and developmental processes. The majority of miRNAs exist intracellularly, while some exist in body fluids, including a variety of extracellular biologic fluids, such as blood, urine, saliva, pancreatic juice, and breast milk (Weber et al., 2010). Under physiological and pathophysiological conditions, exosomes are released through two slightly different mechanisms of "unconventional" exocytosis into the extracellular milieu by several cell types. Whether the packing of miRNAs into exosomes takes place at the pre- or mature-miRNA level has not yet been fully clarified. It has been demonstrated that the sequence motifs present in mature miRNAs can control their sorting into exosomes, and the ubiquitous hnRNPA2B1 recognizing these motifs binds exosomal miRNAs and specifically controls their loading into exosomes (Villarroya-Beltri et al., 2013).

miRNAs dysregulation associated with cancer progression, is common in all human cancers, including OSCC (Lee et al., 2008; Tahiri et al., 2014). Therefore, miRNAs have the potential to be used for the diagnosis and treatment of OSCC (Xu et al., 2016; Božinović et al., 2019; Kirave et al., 2020). miRNAs can be packed into microparticles and exosomes, resulting in transfer of miRNAs to body fluids by a passive release mechanism (Chen et al., 2008; Chim et al., 2008). Exosomes can also be released by tumor cells or circulating microvesicles via shedding as an active secretion mechanism (Shah and Calin, 2013). Exosomal miRNAs from the TME exert diverse effects on tumorigenesis, including the regulation of host immune responses, tumor growth, angiogenesis, metastasis, tumor chemoresistance, and control of the TME. Exosomal miRNAs derived from tumor cells affect the immune activity of several tumor-associated immune cells as well as the transport of signaling molecules among tumor cells, immune cells, and other cell types.

\section{Exosomal miRNAs and OSCC Progression}

The contemporary therapies for OSCC yet are inefficient due to the limited understanding of their underlying mechanisms and the difficulties posed for developing accurate diagnostic methods. As important genetic materials transported in exosomes, exosomal miRNAs could serve as potential biomarkers and therapeutic targets for the treatment of OSCC. MiR-24-3p from salivary exosomes has been reported as a potential biomarker for OSCC (He et al., 2020). Furthermore, exosomal miRNAs play an essential role in growth, metastasis, and drug resistance. Exosomal miR-382-5p and miR-34a-5p from CAFs influence the proliferation, migration, and invasion of OSCC (Li et al., 2018; Sun et al., 2019). Li et al. (2018) showed miR-34a targeting AXL through AKT/GSK-3 $\beta / \beta$-catenin pathway to promote the OSCC progression. Liu et al. (2017) first acquired cisplatin-resistant
OSCC cells and used the conditional medium from resistant cells to treat parent OSCC cells. They further revealed that cisplatin-resistant OSCC cells could transfer miR-21 by exosomes targeting PTEN and PDCD4 to confer the cisplatin-resistance of the parental OSCC cells (Liu et al., 2017). Thus, exosomes may have the function as a vector for resistance transfer in cancer cells, and the resistance-related factors should be considered as therapeutic targets for effective treatment of OSCC.

Differential miRNA contents in OSCC exosomes have been reported in both pre-clinical and clinical studies. Recently, Rabinowits et al. (2017) compared the miRNA content of OSCCderived exosomes with matching benign tissue and plasma from a patient. They found seven downregulated and nine upregulated miRNAs in tumor tissue compared with adjacent tissues. Furthermore, OSCC cells secreted miR-24-3p, miR-891a, miR-106a-5p, miR-2a-5p, and miR-1908 decreases the T-cell response in the tumor stroma by targeting the Mark1 signaling pathway and subsequently manipulating the proliferation and differentiation of cells (Ye et al., 2014). Moreover, miR-142-3p derived from exosomes were found to reduce TGFBR1 activity and promote OSCC cell proliferation in vitro and in vivo (Dickman et al., 2017). Exosomal miR-29a-3p derived from OSCC cells enhances tumor growth in a nude mouse model and M2 macrophage polarization by targeting the SOCS1 (Cai et al., 2019). Tachibana et al. (2016) and Xie et al. (2019a) showed that miR-223 and miR-101-3p function as tumor suppressors by inhibiting cell proliferation and inducing apoptosis through the process of exosome secretion, and exosomes secreting miR338 from OSCC cells were also identified as tumor suppressors. Moreover, it was demonstrated that the overexpression of miR$34 a-5 p$ suppresses the proliferation of both CAL-27 and SCC15 cells (Rabinowits et al., 2017). In addition, based on the colony formation assay, exosomal miR-34a-5p overexpression significantly reduced the colony counts of both CAL-27 and SCC-15 cells (Li et al., 2018).

Increased miRNA expression in exosomes is believed to promote OSCC metastasis. Leukoplakia is a precancerous lesion in OSCC, and it was found that miR-21 secreted from OSCC cells was correlated with low expression of its target genes, TPM1 and PTEN, and was highly expressed in progressive leukoplakia and OSCC to promote disease progression (Liu et al., 2017). Similarly, the involvement of exosome-delivered miRNAs in OSCC metastasis has been reported. Further analysis of six selected miRNAs revealed that miR-200c-3p silences its targets, CHD9 and WRN, as a key exosomal miRNA to promote tumor invasion that significantly accelerates the invasive potential of OSCC cells (Kawakubo-Yasukochi et al., 2018). OSCC-derived exosomes may influence cell motility and angiogenesis that, in turn, can influence OSCC progression. Two oncogenic miRNAs, miR-342-3p and miR-1246, are highly expressed in OSCC exosomes, leading to the metastasis of OSCC and increasing cell motility and invasive ability. miR1246 directly targets DENND2D to promote the motility of tumor cells (Sakha et al., 2016). Thus, miRNAs in exosomes may be considered as non-invasive biomarkers for OSCC screening. On the contrary, inhibitory miRNAs may be delivered with exosomes to treat OSCC. 


\section{Exosomal miRNAs and the OSCC Microenvironment}

The TME contains a complex network of non-malignant cells, molecules, structural components, and chemicals that surround cancer cells. Multiple non-malignant cells, including endothelial cells, pericytes, immune cells, and fibroblasts, together with the surrounding ECM, comprise the supportive stroma of the tumor and manipulate the TME. The "seed and soil" hypothesis is widely accepted in the cancer field (Paget, 1989; Fidler and Poste, 2008). The pre-metastatic niche, conceptualized as a fertile soil conducive to the survival and growth of metastatic seeds, consists of diverse cell populations, such as CAFs and various infiltrating immune cells, and non-cell components of the ECM. These niche components influence the fate of disseminated tumor cells in diverse ways, such as cell proliferation and differentiation, and contribute to tumor angiogenesis, invasion, and metastasis ( $\mathrm{Wu}$ et al., 2018; Peltanova et al., 2019). Exosomes have been identified as a crucial means of cell-to-cell communication, involving both near and distant signal transduction. Thus, tumor-derived exosomes can serve as messengers in the tumor environment, creating favorable environment for tumor growth and metastasis (Bae et al., 2018).

Alteration of the TME is the first step in forming a premetastatic niche. As one of the most abundant constituents of the TME, we demonstrated that CAFs perform critical roles during tumor progression and metastasis ( $\mathrm{Vu}$ et al., 2019). miRNAs from cancer-derived exosomes are crucial messengers in the intercommunication between tumor cells and CAFs within the TME. Bovy et al. (2015) demonstrated that exosomes derived from CAFs enhance OSCC cell metastasis. Besides, fibroblasts in the TME "communicate" with tumor cells through the transfer of miRNAs contained in exosomes (Bovy et al., 2015). Li et al. (2018) found that the expression of miR-34a-5p in CAF-derived exosomes was significant, thereby inducing the EMT and reducing expression of the cancer stem marker AXL to facilitate cancer cell metastasis via the AKT-GSK3 $\beta$ - $\beta$-catenin signaling pathway. Furthermore, the miR-34a-5p-AXL axis enhanced nuclear translocation of $\beta$-catenin, thereby inducing transcriptional upregulation of SNAIL, which in turn activated the ECM proteins MMP-2 and MMP-9. Besides, it was found that miR-3188 expression by directly targeting to BCL2, is reduced in exosomes and their parental CAFs from OSCC tissues (Wang et al., 2019b). Sun et al. (2019) discovered that exosomal miR-382$5 \mathrm{p}$ derived from CAFs and NFs upregulates MMP-3, MMP-9, $\mathrm{N}$-cadherin, and $\beta$-catenin in OSCC cells, thus increasing the migration of CAL-27.

The composition and function of the vasculature in the TME exhibits abnormalities, including leakiness, a heterogeneous basement membrane, irregular vessel branching, and poor pericyte coverage. These changes ultimately lead to a hypoxic TME (Hu and Polyak, 2008), and TEX can then be induced to migrate and invade normoxic cells. Li et al. (2016) showed that a hypoxic microenvironment may stimulate OSCC tumors to produce miR-21-rich exosomes, enabling miR-21 to be transported to normoxic regions and drive non-hypoxic cells toward a pro-metastatic phenotype.
The human immune system exerts its defensive functions by innate immunity and adaptive immunity. Innate immunity provides the body with its instinctive defense against the pathogenic infections, while their propagation brings about activation of adaptive immune responses. The infiltration of lymphocytes, including regulatory $\mathrm{T}$ cells (Tregs), MDSCs, and tumor-associated macrophages, is common in OSCC. By dampening the immune response and generating immune tolerance, these lymphocytic cells promote immune evasion by tumor cells. Li et al. (2019b) suggested that exosomal miR21 plays a key role in the regulation of TEX-induced $\gamma \delta$ T-cell function by affecting MDSCs. These authors utilized lenti-miR-138 virus $\gamma \delta \mathrm{T}$ cell-derived exosomes ( $\gamma \delta \mathrm{TDEs})$ as a drug delivery system in the treatment of OSCC (Xie et al., 2019a). Delivery of miR-138 with $\gamma \delta$ TDEs had a synergistic inhibitory effect on CAL-27 cells in vitro (Xie et al., 2019a). In immunocompetent $\mathrm{C} 3 \mathrm{H}$ mice, applying miR-138-rich $\gamma \delta \mathrm{TDE}$ as a form of pre-immunization inhibited the growth of OSCC (Xie et al., 2019a). Using differential fluorescence, miR-101-3p was found to be transferred from donor hBMSCs to recipient TCA8113 cells. By targeting COL10A, the transferred miR101-3p significantly repressed cell invasion and migration ( $\mathrm{Li}$ et al., 2019c). Further evidence showed that the miR-21-5p that was released from CAL27-derived exosomes was taken up by THP1 monocytes, playing a role in activating the NF- $\kappa$ B inflammatory pathway. The delivery of miR-21-5p by exosomes promotes monocyte migration and infiltration, which in turn participates in the promotion of angiogenesis in OSCC (Momen-Heravi and Bala, 2018).

Interestingly, alcohol treatment ( $25 \mathrm{mM}$ for $24 \mathrm{~h}$ ) increases exosome production and alters the subset of oncogenic miRNAs that are specifically enriched in exosomes released from tumor cells (Momen-Heravi and Bala, 2018). Mechanistically, exosome uptake from OSCC cells by monocytes causes activation of the $\mathrm{NF}-\kappa \mathrm{B}$ pathway and establishment of a pro-inflammatory milieu (Momen-Heravi and Bala, 2018). Thus, TEXs promote changes in the microenvironment, such as oxygen reduction and decreased immune responses, this deterioration of the microenvironment exacerbates the progression of OSCC. However, the presence of exosomes from immune cells in the OSCC microenvironment was not reported.

The traditional therapies for treating OSCC are surgery followed by chemotherapy. Due to the complexity of the TME, conventional drug delivery systems fail to transfer chemotherapeutics in an effective concentration to kill cancer cells and are associated with debilitating side effects. Exosomes have the essential characteristics including biocompatibility, noncytotoxicity, low immunogenicity, simple to produce and store, long life span, and high cargo loading capacity (Steinbichler et al., 2019). The small size confers exosomes resistant to lung clearance and passing through the blood-brain barrier effectively (Kawikova and Askenase, 2015). Furthermore, exosomes may be used as specific targeting against cancer cells rather than normal cells by receptors in exosomes (Wang et al., 2016). Mounting evidence has provided insights about the crucial role of exosomal miRNAs in controlling the TME, and these insights could be applied to drug delivery. These miRNAs could 


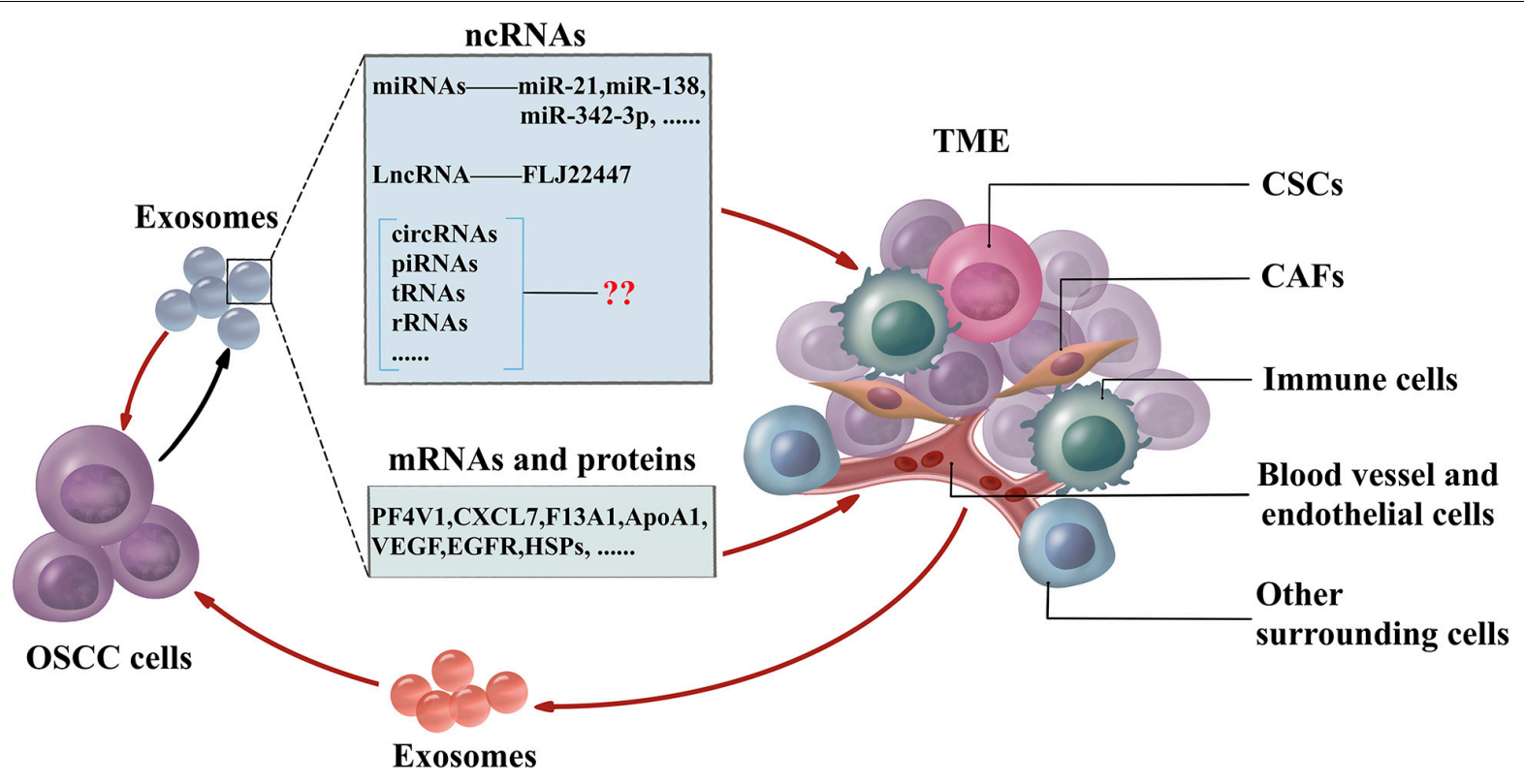

FIGURE 2 | The feedback regulation between OSCC and TME. OSCC cells generate exosomes which include non-coding RNAs (miRNAs, IncRNAs, circRNAs, piRNAs, tsRNAs, and rRNAs), mRNAs, and proteins to influence the TME. CAFs, CSCs, immune cells, blood vessels, endothelial cells, and other surrounding cells are the essential cells in TME. Furthermore, cells in TME influence the function of OSCC cells by exosomes. OSCC cells also interact with adjacent OSCC cells via exosomes. OSCC, oral squamous cell carcinoma; TME, tumor microenvironment; miRNAs, microRNAs; IncRNAs, long non-coding RNAs; circRNAs, circular RNAs; piRNAs, PIWI-interacting RNAs; tRNAs, transfer RNAs; rRNAs, ribosomal RNAs; CAFs, cancer-associated fibroblasts; CSCs, cancer stem cells.

be utilized as therapeutic components delivered to the OSCC microenvironment by exosomes. Exosome-delivered tumor suppressor miRNAs, miR-143 inhibits the growth of prostate cancer, while the let-7a significantly reduces the growth of breast cancer in vivo, respectively (Kosaka et al., 2012; Ohno et al., 2013 b). Bio-safety has also been confirmed by adverse effects detection in normal prostatic epithelial cells with treatment of exosome-containing miR-143 (Kosaka et al., 2012). Cancer may acquire the drug resistance against chemotherapeutics by drug efflux with transporters. Exosomes could deliver the anti-miR-9 to reduce the transporter level, thus, sensitizes glioblastoma cells to temzolomid to increase cell death (Munoz et al., 2013). The feedback regulation of exosomes and TMEs is shown in Figure 2.

\section{EXOSOMAL IncRNAs, CIRCULAR RNAs, AND piRNAs IN OSCC}

Aside from miRNAs, ncRNAs also consist of long non-coding RNAs (lncRNAs), circular RNAs (circRNAs), and piRNAs, rRNAs and tsRNAs. IncRNAs are defined as transcriptional products, with a length of more than 200 nucleotides and generally having no protein-coding potential (Jin et al., 2016). However, ncRNAs involve in multiple pathological processes. Gastrointestinal cancer has the same problem as OSCC of resistance to chemotherapy agents, such as cisplatin.

\section{Exosomal IncRNAs}

Exosomal lncRNAs mediates the progression and chemoresistance of tumor cells in the TME through diverse mechanisms. The exosomal lncRNA HOTTIP, transmitted from cisplatin-resistant gastric cancer cells to sensitive cancer cells, plays a role in conferring cisplatin resistance to sensitive cancer cells by binding to miR-218 to activate HMGA1 (Wang et al., 2019a). Moreover, this lncRNA is upregulated in the bodily fluids of gastric cancer patients, which indicates that it is a potential biomarker for early diagnosis and treatment. Similarly, Kogure et al. (2013) reported that the exosomal lncRNA TUC339 secreted by hepatocellular cancer cells is absorbed by surrounding cells and thereby promotes the growth of hepatocellular carcinoma. Ding et al. (2018) found that the lncRNA FLJ22447 derived from OSCC exosomes is upregulated in CAFs and activates them to induce the proliferation of OSCC cells through IL-33.

\section{Exosomal CircRNAs}

CircRNAs are a kind of single-stranded RNA that is comprised of mostly cytoplasmic exonic particles with linked $3^{\prime}$ and $5^{\prime}$ ends in eukaryotic cells. Several circRNAs have been found in body fluids, such as blood and saliva (Holdt et al., 2018; de Fraipont et al., 2019). circRNAs sorting into exosomes may be controlled by modulation of associated miRNA levels in parental cells and may transfer biological activity to target cells (Li et al., 2020). A recent study has reported that the exosomal circRNAs DB, derived from adipocytes, promotes the growth of hepatocellular carcinoma by sponging miR-34a and activating USP7/Cyclin A2 signaling pathway (Zhang et al., 2019). Zhao et al. (2019) found that the circRNA ATP8B4 acts as miR-766 sponge and plays a role in the development of radiation resistance in glioblastoma. The circRNA CDR1as functions as a miR-7 sponge, regulating insulin 
transcription and secretion in pancreatic islet cells via miR-7 targets and downregulates miR-7 to perturb the development of diabetes (Xu et al., 2015). Hansen et al. (2013) introduced miR-7 mimics into HEK293T and MCF-7 cell lines and reported that the level of exosomal CDR1as is significantly decreased while increased in cells due to the ectopic expression of miR-7 in both HEK293T and MCF-7 cells.

\section{Exosomal piRNAs}

piRNAs are abundant small, non-coding, single-stranded RNAs 21-30 nucleotides in length, with little conservation of sequence between organisms (Weick and Miska, 2014). Compared with miRNAs, piRNAs function to repress transposons at the transcriptional and posttranscriptional levels to maintain genome integrity, while miRNAs play a role in repressing translation at the post-transcriptional level to regulate gene expression (Iwasaki et al., 2015). Although piRNAs have only $1-4 \%$ of all identified sequence content in the exosome, it was found that piRNAs are as abundant as miRNAs in exosomes isolated from plasma (Yuan et al., 2016). piRNAs are regarded as potential biomarkers in breast cancer, colon, and gastric cancer for pathological expression (Cheng et al., 2011; Hashim et al., 2014; Vychytilova-Faltejskova et al., 2018). Mounting evidence shows that piRNAs strongly correlate with tumor cell malignant phenotype and clinical stage. piR-021285 regulates cell proliferation and invasion by DNA methylation. The piR021285 variant mimics transfection into breast cancer cell lines and weakens pro-invasive and pro-apoptosis gene methylation of ARHGAP11A at the 5'-UTR-first exon CpG site, which results in higher ARHGAP11A expression and increased breast cancer cell invasiveness (Fu et al., 2015). piRNAs are also involved in the development of lung cancer. The tumor promoter RASSF1C upregulates piR-34871 and piR-52200 and downregulates piR35127 and piR-46545 through the RASSF1C-PIWIL1-piRNA axis, resulting in the promotion of stem cell proliferation and the EMT in lung cancer. These piRNAs changes inhibit AMPK phosphorylation in the ATM-AMPK-p53-p21cip pathway and thereby block cell cycle arrest and enhance cell proliferation (Reeves et al., 2012). Moreover, we developed chemical induced OSCC mouse model and found some piRNAs were significantly changed. And piRNAs has been identified in the exosomes of mesenchymal stem cells (Wang et al., 2020). However, the exosomal function of piRNAs in human OSCC should be further revealed.

Generally, exosomal lncRNAs, circRNAs, and piRNAs are involved in the tumor development. However, there are few reports on the involvement of these exosomal ncRNAs in the functioning of OSCC and their potential as biomarkers in OSCC, which deserves more attention and deeper exploration.

\section{mRNAS AND PROTEINS IN OSCC}

Proteins contained inside exosomes have also been evaluated in patients with OSCC, although not as extensively as miRNAs, and studies have shown promising exosomal protein markers for early diagnosis of OSCC, such as TRAP1, EGFR, heat shock protein 90 (HSP-90), and MMP-13, which can affect the intracellular functions of genes (Kaskas et al., 2014; Xie et al., 2019b). Among the proteins contained in exosomes, 23 were identified as potential biomarkers of OSCC (Boldrup et al., 2017). Recent studies found that the contents of free exosomes in blood were correlated with OSCC cells. Proteins in those exosomes, including PF4V1, CXCL7, F13A1, and ApoA1, could be used in the diagnosis of OSCC ( $\mathrm{Li}$ et al., 2019a). Angiogenesis is generally correlated with tumor growth and metastasis, and exosomes derived from OSCC cells could have an inhibitory or promotional effect on angiogenesis, thereby influencing OSCC metastasis (Zhang et al., 2019). Rosenberger et al. (2019) demonstrated that exosomes manipulate the secretion of VEGF to inhibit the angiogenic activity of endothelial cells, thus reducing tumor metastasis. It was found that exosome treatment inhibits angiogenic activity, including both vessel density and vascular area.

The EMT also plays an essential role in tumor migration and invasion. Overexpression of EGFR is an essential feature of OSCC. It was found that OSCC cells abundantly express EGFR, which is secreted from cells as OSCC exosomes upon EGF stimulation (Fujiwara et al., 2018). Furthermore, OSCC LNM was always found in patients who were diagnosed in the later stages of the disease. Li et al. (2019a) isolated exosomes from the serum of OSCC patients, and found that the exosomal proteins PF4V1, CXCL7, F13A1, and ApoA1 in serum affect OSCC LNM and thereby influence prognosis. ROC analysis is a kind quantification method to acquire desirable levels of sensitivity and specificity (Obuchowski and Bullen, 2018). ROC analysis using the relative abundances of ApoA1, CXCL7, PF4V1, and F13A1 in serum, serum exosomes, and whole blood indicated that exosomal proteins are potentially predictive biomarkers for OSCC with LNM.

Cancer cells often secrete exosomes carrying heat shock proteins, which play a part in tumor progression. It was found that abundant secretion of exosomes rich in HSP-90 was found in OSCC with LNM, indicating a poor prognosis (Ono et al., 2018). Besides, drug resistance also remains a severe problem in most chemotherapy treatments for OSCC (Samuel et al., 2017). Recently, it has been suggested that cancer cell-derived exosomes mediate drug resistance. Khoo et al. (2019) showed the increased exosome production in both de novo (H314) and adaptive (H103/cisD2) resistant cell lines compared with sensitive H103 cells. Moreover, differences in the proteomes contained within exosomes indicate that adaptation to cisplatin treatment causes significant changes in the secreted exosomes (Khoo et al., 2019). The cargos in the exosomes were showed in Table 1.

\section{DISCUSSION}

In 2012, miRNAs were found in the exosomes of invasive tumors, suggesting that tumor-derived exosomes may serve as an important diagnostic tool to avoid metastasis and improve prognosis (Chen et al., 2012a). Therefore, it is essential to determine the involvement of exosomes in maintaining the 
aggressive phenotype of OSCC cells and their unique roles in intercellular communication.

Although several miRNAs have been identified in the exosomes of OSCC, more should be analyzed by improving the isolation and purification of exosomes. Furthermore, the function of IncRNAs, circRNAs, piRNAs, mRNAs, and proteins in the exosomes of OSCC also needs to be determined. In fact, tRNAs and rRNAs are infrastructural ncRNAs, they are also involved into the cancer initiation and progression. tRNAs re small conserved RNA molecules that allow the translation of the genetic code into amino acids. Overexpression of initiator $\mathrm{tRNA}^{\mathrm{Met}}\left(\mathrm{tRNA}_{i}^{\mathrm{Met}}\right)$ is to promote metastasis of melanoma cells, through $\alpha 5 \beta 1$ integrindependent signaling (Birch et al., 2016). Furthermore, the

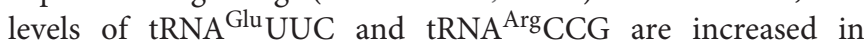
metastatic breast cancer cell lines (Goodarzi et al., 2016). Dysregulated rRNA transcription is essential in cancers and may be involved in the initiation stage of hepatocarcinogenesis (Donati et al., 2011; Xie et al., 2018). However, whether rRNAs are existed in exosomes and the exosomal rRNAs should be further revealed.

As a viable alternative to tissue-based sampling in the clinic, it is clear that there is a great deal of interest in noninvasive liquid biopsies (Xue et al., 2019). Liquid biopsies utilize blood and saliva to detect the circulating tumor cells, circulating tumor DNA, and exosomes for the diagnosis and prognosis of oral cancer (Lousada-Fernandez et al., 2018). This method allows repeated sampling to monitor the treatment response, assess tumor heterogeneity, and even use in cancer screening programs. Wu et al. (2017) has shown that an acoustofluidic platform integrating acoustics and microfluidics efficiently isolates exosomes directly from undiluted blood samples and saliva. Saliva collection was rather easy and noninvasive. It has demonstrated that exosomes have a higher amount and larger size in the saliva of patients with oral cancer than healthy individuals (Zlotogorski-Hurvitz et al., 2016; Nair et al., 2018). Furthermore, increased expression of CD63 and lesser expression of CD9 and CD81 are found in the saliva exosomes of oral cancer patients (Zlotogorski-Hurvitz et al., 2016). More efforts should be made to identify new biomarkers in the exosomes of saliva, finally increasing the application in non-invasive cancer diagnosis. Considering the endogenous transport function, exosomes have robust potential to be applied as therapeutic delivery systems. However, efficiency in delivering drugs to the tumor is still a major challenge due to the blood-brain barrier and degradation (Kawikova and Askenase, 2015). Exosomes can be fused with the cell membrane with high reliability, and therefore non-coding RNAs or peptide drugs may be packed into exosomes and delivered to the OSCC tumor. Furthermore, the process of exosomes transfected with ncRNAs may influence the activity of exosomes. Thus, ncRNAs could be transfected into the exosome derived cells. It has demonstrated exosomes from mesenchymal stem cells overexpressed with tumor suppressed miRNAs has inhibitory effect on tumor progression (Che et al., 2019; Xu et al., 2019; Yuan et al., 2019). Furthermore, the fabrication of target-specific exosomes could increase the efficacy of cancer treatment (Wang et al., 2016). The exosomes conjugated with antibodies specifically target cancer cells (Stickney et al., 2016). The exosomes magnetized or modified by $\mathrm{pH}$-sensitive peptide also contributes to the accumulation in the cancer cells (Nakase and Futaki, 2015; Qi et al., 2016). Furthermore, the glycosylation of the surface proteins increases the stability of exosomes (Hung and Leonard, 2015). The therapeutic application of exosomes against OSCC is illustrated in Figure 3.

In summary, it is essential to understand the role of exosomes in influencing tumor phenotype, angiogenesis,

TABLE 1 | Cargos in exosomes derived from OSCC.

\begin{tabular}{|c|c|c|c|c|}
\hline Biomolecules & Function & Molecules & Exosome origin & References \\
\hline \multirow[t]{7}{*}{ miRNAs } & Suppressor & $\begin{array}{l}\text { miR-338, miR-24-3p, miR-891a, } \\
\text { miR-106a-5p, miR-2a-5p, } \\
\text { miR-1908, miR-101-3p }\end{array}$ & Cell lines & $\begin{array}{l}\text { Ye et al., 2014; Tachibana et al., } \\
\text { 2016; Xie et al., 2019a }\end{array}$ \\
\hline & Biomarker & miR-223 & Plasma & Tachibana et al., 2016 \\
\hline & Metastasis & $\begin{array}{l}\text { miR-21, miR-142-3p, miR-29a-3p, } \\
\text { miR-342-3p, miR-1246, } \\
\text { miR-200c-3p }\end{array}$ & Cell lines & $\begin{array}{l}\text { Sakha et al., 2016; Dickman et al., } \\
\text { 2017; Liu et al., 2017; } \\
\text { Kawakubo-Yasukochi et al., 2018; } \\
\text { Cai et al., } 2019\end{array}$ \\
\hline & Accumulation of fibronectin & $\begin{array}{l}\text { miR-382-5p, miR-34a-5p, } \\
\text { miR-3188 }\end{array}$ & CAFs & $\begin{array}{l}\text { Bovy et al., 2015; Liu et al., 2017; } \\
\text { Li et al., } 2018\end{array}$ \\
\hline & Motility & miR-342-3p, miR-1246, & Cell lines & Sakha et al., 2016 \\
\hline & Angiogenesis & $\operatorname{miR}-21$ & Cell lines & Momen-Heravi and Bala, 2018 \\
\hline & Immune regulation & miR-138, & Cell lines & Xie et al., 2019a \\
\hline \multirow[t]{2}{*}{ Proteins } & Biomarker & PF4V1, CXCL7, F13A1, ApoA1 & Serum & Li et al., 2019a \\
\hline & & VEGF, EGFR, HSPs & OSCC cells & $\begin{array}{l}\text { Fujiwara et al., 2018; Ono et al., } \\
\text { 2018; Rosenberger et al., } 2019\end{array}$ \\
\hline IncRNAs & Proliferation & Lnc-FLJ22447 & OSCC cells & Ding et al., 2018 \\
\hline
\end{tabular}

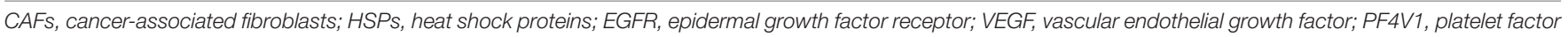
4 variant 1; CXCL7, C-X-C motif chemokine ligand 7; F13A1, coagulation factor XIII A chain; ApoA1, apolipoprotein A1. 


\section{A}

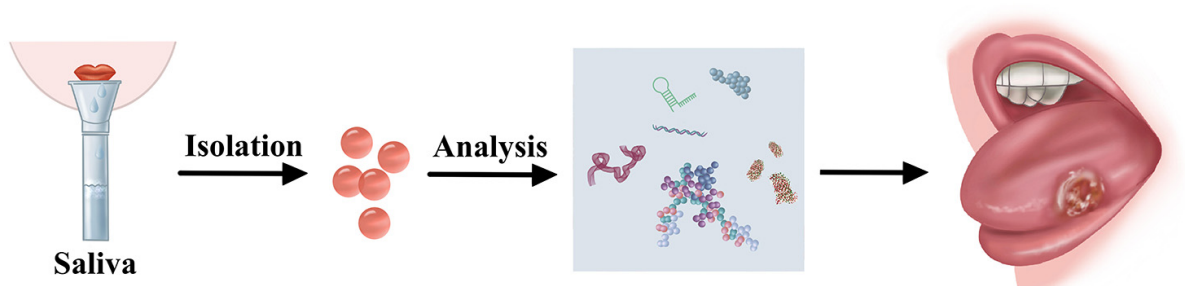

Noninavasive

Exosomes

Biomarks

Noninvasive OSCC

liquid biopsy

molecular diagnosis

B

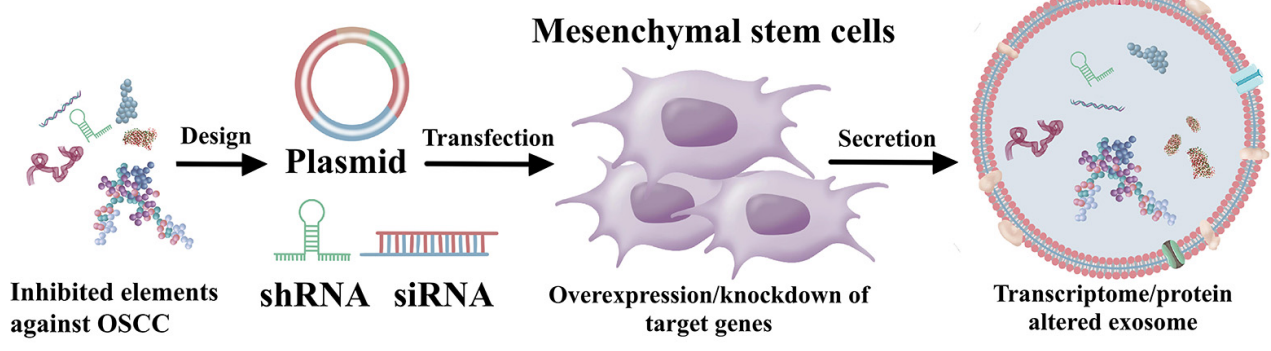

against OSCC

target genes

C

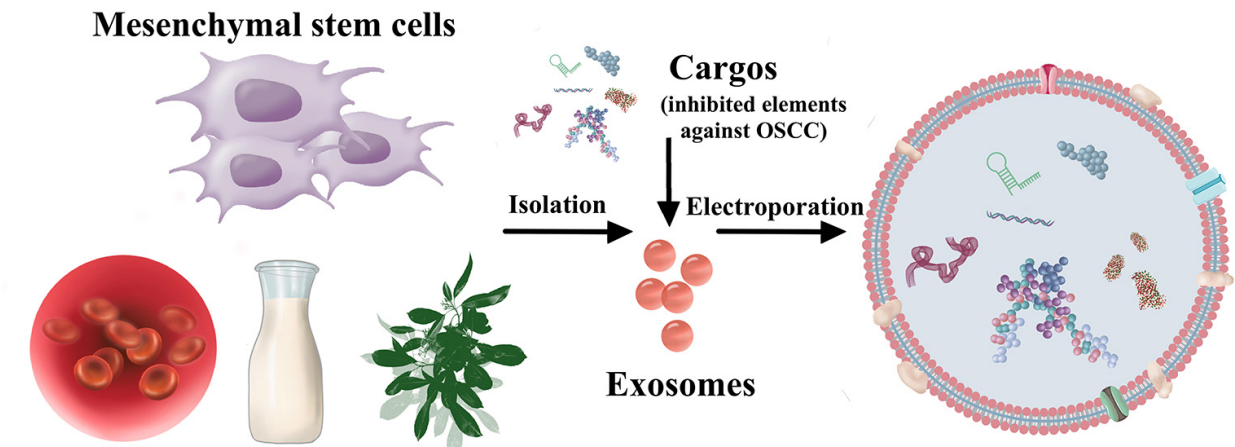

Blood Milk Plant

Cargo-loaded exosomes

D
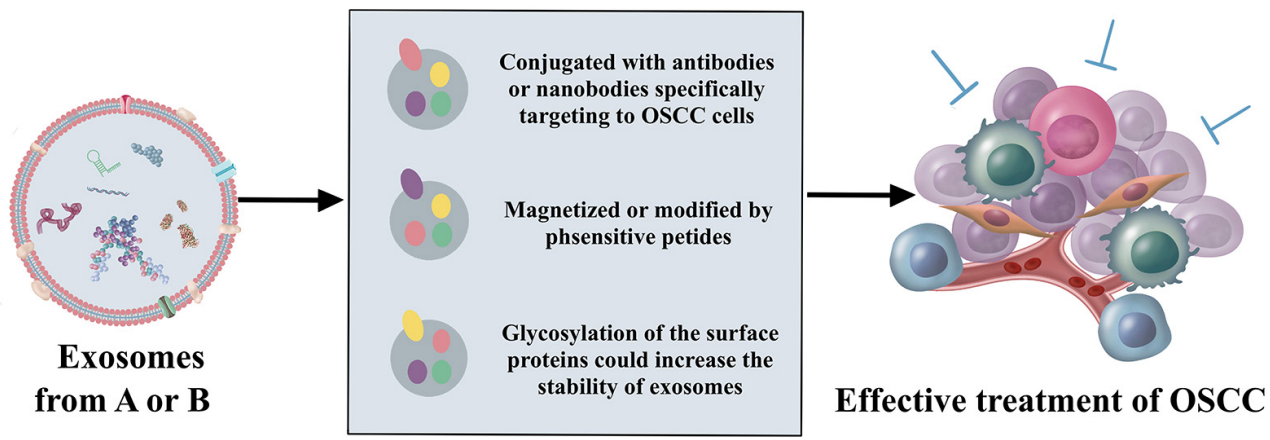

Target specific exosomes

\section{Effective treatment of OSCC}

FIGURE 3 | The summary of therapeutic application of exosomes. (A) The non-invasive diagnosis and prognosis of OSCC using exosomes. (B) Approaches to alter cargo contents in the mesenchymal stem cell-derived exosomes to treat OSCC. (C) Loading of exogenous proteins, genes, or signaling molecules in the exosomes to treat OSCC. (D) Exosome surface modification for target specific delivery of exosomes toward OSCC. 
immune modulation, metastasis, and drug resistance. While studies on the role of exosomes in OSCC have made progress, there are several outstanding questions that need to be further explored. The versatile biological functions of exosomes could be promising tools to apply in the diagnosis, prognosis, and effective treatment of OSCC.

\section{AUTHOR CONTRIBUTIONS}

$\mathrm{LWu}, \mathrm{MZ}$, and WC concepted the manuscript. YL, ZZ, and YY wrote the original draft. JP, XY, LWa, LWu, and WC revised the manuscript. $\mathrm{ZY}$ made the improvement for the

\section{REFERENCES}

Abak, A., Abhari, A., and Rahimzadeh, S. (2018). Exosomes in cancer: small vesicular transporters for cancer progression and metastasis, biomarkers in cancer therapeutics. PeerJ 6:e4763. doi: 10.7717/peerj.4763

Adel, M., Liao, C. T., Lee, L. Y., Hsueh, C., Lin, C. Y., Fan, K. H., et al. (2016). Incidence and outcomes of patients with oral cavity squamous cell carcinoma and fourth primary tumors: a long-term follow-up study in a betel quid chewing endemic area. Medicine (Baltimore) 95:e2950. doi: 10.1097/md 0000000000002950

Ahmadi, M., and Rezaie, J. (2020). Tumor cells derived-exosomes as angiogenenic agents: possible therapeutic implications. J. Transl. Med. 18:249. doi: 10.1186/ s12967-020-02426-5

Amiri, A., Pourhanifeh, M. H., et al. (2021). Exosomes and lung cancer: roles in pathophysiology, diagnosis and therapeutic applications. Curr. Med. Chem. 28, 308-328. doi: 10.2174/0929867327666200204141952

Asgarpour, K., Shojaei, Z., Amiri, F., Ai, J., Mahjoubin-Tehran, M., Ghasemi, F., et al. (2020). Exosomal microRNAs derived from mesenchymal stem cells: cellto-cell messages. Cell Commun. Signal. 18:149. doi: 10.1186/s12964-020-00650-

Azmi, A. S., Bao, B., and Sarkar, F. H. (2013). Exosomes in cancer development, metastasis, and drug resistance: a comprehensive review. Cancer Metastasis Rev. 32, 623-642. doi: 10.1007/s10555-013-9441-9

Bae, S., Brumbaugh, J., and Bonavida, B. (2018). Exosomes derived from cancerous and non-cancerous cells regulate the anti-tumor response in the tumor microenvironment. Genes Cancer 9, 87-100. doi: 10.18632/genesandcancer.172

Bartel, D. P. (2004). MicroRNAs: genomics, biogenesis, mechanism, and function. Cell 116, 281-297. doi: 10.1016/s0092-8674(04)00045-5

Beach, A., Zhang, H. G., Ratajczak, M. Z., and Kakar, S. S. (2014). Exosomes: an overview of biogenesis, composition and role in ovarian cancer. J. Ovarian Res. 7:14. doi: 10.1186/1757-2215-7-14

Birch, J., Clarke, C. J., Campbell, A. D., Campbell, K., Mitchell, L., Liko, D., et al. (2016). The initiator methionine tRNA drives cell migration and invasion leading to increased metastatic potential in melanoma. Biol. Open 5, 1371-1379. doi: 10.1242/bio.019075

Boldrup, L., Troiano, G., Gu, X., Coates, P., Fåhraeus, R., Wilms, T., et al. (2017). Evidence that circulating proteins are more promising than miRNAs for identification of patients with squamous cell carcinoma of the tongue. Oncotarget 8, 103437-103448. doi: 10.18632/oncotarget.21402

Bovy, N., Blomme, B., Frères, P., Dederen, S., Nivelles, O., Lion, M., et al. (2015). Endothelial exosomes contribute to the antitumor response during breast cancer neoadjuvant chemotherapy via microRNA transfer. Oncotarget 6, 10253-10266. doi: 10.18632/oncotarget.3520

Božinović, K., Sabol, I., Dediol, E., Milutin Gašperov, N., Manojlović, S., Vojtechova, Z., et al. (2019). Genome-wide miRNA profiling reinforces the importance of miR-9 in human papillomavirus associated oral and oropharyngeal head and neck cancer. Sci. Rep. 9:2306. doi: 10.1038/s41598-01938797-z

Bu, J., Bu, X., Liu, B., Chen, F., and Chen, P. (2015). Increased expression of tissue/salivary transgelin mRNA predicts poor prognosis in patients with Oral figures. All authors contributed to the article and approved the submitted version.

\section{FUNDING}

This work was supported by the National Natural Science Foundation of China (31801152), the Innovation and Entrepreneurship Training Program for College Students of Guangdong (S201910570039), and the University Student Laboratory Open Project of Guangzhou Medical University (2020-27).

Squamous Cell Carcinoma (OSCC). Med. Sci. Monit. 21, 2275-2281. doi: 10 $12659 / \mathrm{msm} .893925$

Cai, J., Qiao, B., Gao, N., Lin, N., and He, W. (2019). Oral squamous cell carcinomaderived exosomes promote M2 subtype macrophage polarization mediated by exosome-enclosed miR-29a-3p. Am. J. Physiol. Cell Physiol. 316, C731-C740. doi: 10.1152/ajpcell.00366.2018

Cao, J., Zhang, M., Xie, F., Lou, J., Zhou, X., Zhang, L., et al. (2020). Exosomes in head and neck cancer: roles, mechanisms and applications. Cancer Lett. 494, 7-16. doi: 10.1016/j.canlet.2020.07.005

Che, Y., Shi, X., Shi, Y., Jiang, X., Ai, Q., Shi, Y., et al. (2019). Exosomes derived from miR-143-Overexpressing MSCs inhibit cell migration and invasion in human prostate cancer by downregulating TFF3. Mol. Ther. Nucleic Acids 18, 232-244. doi: 10.1016/j.omtn.2019.08.010

Chen, X., Ba, Y., Ma, L., Cai, X., Yin, Y., Wang, K., et al. (2008). Characterization of microRNAs in serum: a novel class of biomarkers for diagnosis of cancer and other diseases. Cell Res. 18, 997-1006. doi: 10.1038/cr.2008.282

Chen, X., Liang, H., Zhang, J., Zen, K., and Zhang, C. Y. (2012a). Horizontal transfer of microRNAs: molecular mechanisms and clinical applications. Protein Cell 3, 28-37. doi: 10.1007/s13238-012-2003-Z

Chen, X., Liang, H., Zhang, J., Zen, K., and Zhang, C. Y. (2012b). Secreted microRNAs: a new form of intercellular communication. Trends Cell Biol. 22, 125-132. doi: 10.1016/j.tcb.2011.12.001

Cheng, J., Guo, J. M., Xiao, B. X., Miao, Y., Jiang, Z., Zhou, H., et al. (2011). piRNA, the new non-coding RNA, is aberrantly expressed in human cancer cells. Clin. Chim. Acta 412, 1621-1625. doi: 10.1016/j.cca.2011.05.015

Chim, S. S., Shing, T. K., Hung, E. C., Leung, T. Y., Lau, T. K., Chiu, R. W., et al. (2008). Detection and characterization of placental microRNAs in maternal plasma. Clin. Chem. 54, 482-490. doi: 10.1373/clinchem.2007.09 7972

Coyte, A., Morrison, D. S., and McLoone, P. (2014). Second primary cancer risk the impact of applying different definitions of multiple primaries: results from a retrospective population-based cancer registry study. BMC Cancer 14:272. doi: 10.1186/1471-2407-14-272

Csõsz, É, Lábiscsák, P., Kalló, G., Márkus, B., Emri, M., Szabó, A., et al. (2017) Proteomics investigation of OSCC-specific salivary biomarkers in a Hungarian population highlights the importance of identification of populationtailored biomarkers. PLoS One 12:e0177282. doi: 10.1371/journal.pone.017 7282

de Fraipont, F., Gazzeri, S., Cho, W. C., and Eymin, B. (2019). Circular RNAs and RNA splice variants as biomarkers for prognosis and therapeutic response in the liquid biopsies of Lung Cancer patients. Front. Genet. 10:390. doi: 10.3389/ fgene. 2019.00390

Dickman, C. T., Lawson, J., Jabalee, J., MacLellan, S. A., LePard, N. E., Bennewith K. L., et al. (2017). Selective extracellular vesicle exclusion of miR-142-3p by oral cancer cells promotes both internal and extracellular malignant phenotypes. Oncotarget 8, 15252-15266. doi: 10.18632/oncotarget.14862

Ding, L., Ren, J., Zhang, D., Li, Y., Huang, X., Hu, Q., et al. (2018). A novel stromal lncRNA signature reprograms fibroblasts to promote the growth of oral squamous cell carcinoma via LncRNA-CAF/interleukin-33. Carcinogenesis 39, 397-406. doi: 10.1093/carcin/bgy006 
Donati, G., Bertoni, S., Brighenti, E., Vici, M., Trere, D., Volarevic, S., et al. (2011). The balance between rRNA and ribosomal protein synthesis up- and downregulates the tumour suppressor p53 in mammalian cells. Oncogene 30, 3274-3288. doi: 10.1038/onc.2011.48

Doyle, L. M., and Wang, M. Z. (2019). Overview of extracellular vesicles, their origin, composition, purpose, and methods for exosome isolation and analysis. Cells 8:727. doi: 10.3390/cells8070727

Fidler, I. J., and Poste, G. (2008). The "seed and soil" hypothesis revisited. Lancet Oncol. 9:808. doi: 10.1016/s1470-2045(08)70201-8

Fu, A., Jacobs, D. I., Hoffman, A. E., Zheng, T., and Zhu, Y. (2015). PIWIinteracting RNA 021285 is involved in breast tumorigenesis possibly by remodeling the cancer epigenome. Carcinogenesis 36, 1094-1102. doi: 10.1093/ carcin/bgv105

Fujiwara, T., Eguchi, T., Sogawa, C., Ono, K., Murakami, J., Ibaragi, S., et al. (2018). Carcinogenic epithelial-mesenchymal transition initiated by oral cancer exosomes is inhibited by anti-EGFR antibody cetuximab. Oral. Oncol. 86, 251-257. doi: 10.1016/j.oraloncology.2018.09.030

Gai, C., Camussi, F., Broccoletti, R., Gambino, A., Cabras, M., Molinaro, L., et al. (2018). Salivary extracellular vesicle-associated miRNAs as potential biomarkers in oral squamous cell carcinoma. BMC Cancer 18:439. doi: 10.1186/ s12885-018-4364-Z

Ghaemmaghami, A. B., Mahjoubin-Tehran, M., Movahedpour, A., Morshedi, K., Sheida, A., Taghavi, S. P., et al. (2020). Role of exosomes in malignant glioma: microRNAs and proteins in pathogenesis and diagnosis. Cell Commun. Signal. 18:120. doi: 10.1186/s12964-020-00623-9

Goodarzi, H., Nguyen, H. C. B., Zhang, S., Dill, B. D., Molina, H., et al. (2016). Modulated expression of specific tRNAs drives gene expression and cancer progression. Cell 165, 1416-1427. doi: 10.1016/j.cell.2016.05.046

Hansen, T. B., Kjems, J., and Damgaard, C. K. (2013). Circular RNA and miR-7 in cancer. Cancer Res. 73, 5609-5612. doi: 10.1158/0008-5472.CAN-13-1568

Hashemian, S. M., Pourhanifeh, M. H., Fadaei, S., Velayati, A. A., Mirzaei, H., and Hamblin, M. R. (2020). Non-coding RNAs and exosomes: their role in the pathogenesis of sepsis. Mol. Therapy-Nucleic Acids 21, 51-74. doi: 10.1016/j. omtn.2020.05.012

Hashim, A., Rizzo, F., Marchese, G., Ravo, M., Tarallo, R., Nassa, G., et al. (2014). RNA sequencing identifies specific PIWI-interacting small non-coding RNA expression patterns in breast cancer. Oncotarget 5, 9901-9910. doi: 10.18632/ oncotarget. 2476

He, L., Ping, F., Fan, Z. N., Zhang, C., Deng, M., Cheng, B., et al. (2020). Salivary exosomal miR-24-3p serves as a potential detective biomarker for oral squamous cell carcinoma screening. Biomed. Pharmacotherapy 121:109553. doi: 10.1016/j.biopha.2019.109553

Holdt, L. M., Kohlmaier, A., and Teupser, D. (2018). Molecular roles and function of circular RNAs in eukaryotic cells. Cell Mol. Life Sci. 75, 1071-1098. doi: 10.1007/s00018-017-2688-5

$\mathrm{Hu}$, M., and Polyak, K. (2008). Microenvironmental regulation of cancer development. Curr. Opin. Genet. Dev. 18, 27-34. doi: 10.1016/j.gde.2007.12.006

Huang, W. C., Jang, T. H., Tung, S. L., Yen, T. C., Chan, S. H., and Wang, L. H. (2019). A novel miR-365-3p/EHF/keratin 16 axis promotes oral squamous cell carcinoma metastasis, cancer stemness and drug resistance via enhancing $\beta 5$ integrin/c-met signaling pathway. J. Exp. Clin. Cancer Res. 38:89. doi: 10.1186/ s13046-019-1091-5

Hung, M. E., and Leonard, J. N. (2015). Stabilization of exosome-targeting peptides via engineered glycosylation. J. Biol. Chem. 290, 8166-8172. doi: 10.1074/jbc. M114.621383

Hwang, H. W., and Mendell, J. T. (2006). MicroRNAs in cell proliferation, cell death, and tumorigenesis. Br. J. Cancer 94, 776-780. doi: 10.1038/sj.bjc.6603023

Iwasaki, Y. W., Siomi, M. C., and Siomi, H. (2015). PIWI-Interacting RNA: its biogenesis and functions. Annu. Rev. Biochem. 84, 405-433. doi: 10.1146/ annurev-biochem-060614-034258

Jabbari, N., Akbariazar, E., Feqhhi, M., Rahbarghazi, R., and Rezaie, J. (2020a). Breast cancer-derived exosomes: tumor progression and therapeutic agents. J. Cell Physiol. 235, 6345-6356. doi: 10.1002/jcp.29668

Jabbari, N., Karimipour, M., Khaksar, M., Akbariazar, E., Heidarzadeh, M., Mojarad, B., et al. (2020b). Tumor-derived extracellular vesicles: insights into bystander effects of exosomes after irradiation. Lasers Med. Sci. 35, 531-545. doi: 10.1007/s10103-019-02880-8
Jiang, Z., Liu, G., and Li, J. (2020). Recent progress on the isolation and detection methods of exosomes. Chem. Asian J. 15, 3973-3982. doi: 10.1002/ asia. 202000873

Jin, C., Shi, W., Wang, F., Shen, X., Qi, J., Cong, H., et al. (2016). Long non-coding RNA HULC as a novel serum biomarker for diagnosis and prognosis prediction of gastric cancer. Oncotarget 7, 51763-51772. doi: 10.18632/oncotarget. 10107

Kalra, H., Simpson, R. J., Ji, H., Aikawa, E., Altevogt, P., Askenase, P., et al. (2012). Vesiclepedia: a compendium for extracellular vesicles with continuous community annotation. PLoS Biol. 10:e1001450. doi: 10.1371/journal.pbio. 1001450

Kaskas, N. M., Moore-Medlin, T., McClure, G. B., Ekshyyan, O., Vanchiere, J. A., and Nathan, C. A. (2014). Serum biomarkers in head and neck squamous cell cancer. JAMA Otolaryngol. Head Neck Surg. 140, 5-11. doi: 10.1001/jamaoto. 2013.5688

Kawakubo-Yasukochi, T., Morioka, M., Hazekawa, M., Yasukochi, A., Nishinakagawa, T., Ono, K., et al. (2018). miR-200c-3p spreads invasive capacity in human oral squamous cell carcinoma microenvironment. Mol. Carcinog. 57, 295-302. doi: 10.1002/mc.22744

Kawikova, I., and Askenase, P. W. (2015). Diagnostic and therapeutic potentials of exosomes in CNS diseases. Brain Res. 1617, 63-71. doi: 10.1016/j.brainres.2014. 09.070

Keller, S., Sanderson, M. P., Stoeck, A., and Altevogt, P. (2006). Exosomes: from biogenesis and secretion to biological function. Immunol. Lett. 107, 102-108. doi: 10.1016/j.imlet.2006.09.005

Khoo, X. H., Paterson, I. C., Goh, B. H., and Lee, W. L. (2019). Cisplatin-Resistance in oral squamous cell carcinoma: regulation by tumor cell-derived extracellular vesicles. Cancers (Basel) 11:1166. doi: 10.3390/cancers11081166

Kim, V. N., Han, J., and Siomi, M. C. (2009). Biogenesis of small RNAs in animals. Nat. Rev. Mol. Cell Biol. 10, 126-139. doi: 10.1038/nrm2632

Kirave, P., Gondaliya, P., Kulkarni, B., Rawal, R., Garg, R., Jain, A., et al. (2020). Exosome mediated miR-155 delivery confers cisplatin chemoresistance in oral cancer cells via epithelial-mesenchymal transition. Oncotarget 11, 1157-1171. doi: 10.18632/oncotarget.27531

Kogure, T., Yan, I. K., Lin, W. L., and Patel, T. (2013). Extracellular vesicle-mediated transfer of a novel long noncoding RNA TUC339: a mechanism of intercellular signaling in human Hepatocellular Cancer. Genes Cancer 4, 261-272. doi: 10. $1177 / 1947601913499020$

Kosaka, N., Iguchi, H., Yoshioka, Y., Hagiwara, K., Takeshita, F., and Ochiya, T. (2012). Competitive interactions of cancer cells and normal cells via secretory microRNAs. J. Biol. Chem. 287, 1397-1405. doi: 10.1074/jbc.M111.28 8662

Lee, J. W., Choi, C. H., Choi, J. J., Park, Y. A., Kim, S. J., Hwang, S. Y., et al. (2008). Altered MicroRNA expression in cervical carcinomas. Clin. Cancer Res. 14, 2535-2542. doi: 10.1158/1078-0432.ccr-07-1231

Leidal, A. M., and Debnath, J. (2020). Unraveling the mechanisms that specify molecules for secretion in extracellular vesicles. Methods 177, 15-26. doi: 10 . 1016/j.ymeth.2020.01.008

Li, C., Zhou, Y., Liu, J., Su, X., Qin, H., Huang, S., et al. (2019d). Potential markers from serum-purified exosomes for detecting oral squamous cell carcinoma metastasis. Cancer Epidemiol. Biomarkers Prev. 28, 1668-1681. doi: 10.1158/ 1055-9965.epi-18-1122

Li, L., Cao, B., Liang, X., Lu, S., Luo, H., Wang, Z., et al. (2019a). Microenvironmental oxygen pressure orchestrates an anti- and pro-tumoral $\gamma \delta \mathrm{T}$ cell equilibrium via tumor-derived exosomes. Oncogene 38, 2830-2843. doi: 10.1038/s41388-018-0627-z

Li, L., Li, C., Wang, S., Wang, Z., Jiang, J., Wang, W., et al. (2016). Exosomes derived from hypoxic oral squamous cell carcinoma cells deliver miR-21 to normoxic cells to elicit a prometastatic phenotype. Cancer Res. 76, 1770-1780. doi: 10.1158/0008-5472.can-15-1625

Li, L., Lu, S., Liang, X., Cao, B., Wang, S., Jiang, J., et al. (2019b). $\gamma \delta$ TDEs: an efficient delivery system for miR-138 with Anti-tumoral and immunostimulatory roles on oral squamous cell carcinoma. Mol. Ther. Nucleic Acids 14, 101-113. doi: 10.1016/j.omtn.2018.11.009

Li, P., Kaslan, M., Lee, S. H., Yao, J., and Gao, Z. (2017). Progress in exosome isolation techniques. Theranostics 7, 789-804. doi: 10.7150/thno. 18133 
Li, R., Jiang, J., Shi, H., Qian, H., Zhang, X., and Xu, W. (2020). CircRNA: a rising star in gastric cancer. Cell Mol. Life Sci. 77, 1661-1680. doi: 10.1007/s00018019-03345-5

Li, X., Corbett, A. L., Taatizadeh, E., Tasnim, N., Little, J. P., Garnis, C., et al. (2019c). Challenges and opportunities in exosome research-Perspectives from biology, engineering, and cancer therapy. APL Bioeng. 3:011503. doi: 10.1063/1. 5087122

Li, Y. Y., Tao, Y. W., Gao, S., Li, P., Zheng, J. M., Zhang, S. E., et al. (2018). Cancerassociated fibroblasts contribute to oral cancer cells proliferation and metastasis via exosome-mediated paracrine miR-34a-5p. EBioMedicine 36, 209-220. doi: 10.1016/j.ebiom.2018.09.006

Liu, T., Chen, G., Sun, D., Lei, M., Li, Y., Zhou, C., et al. (2017). Exosomes containing miR-21 transfer the characteristic of cisplatin resistance by targeting PTEN and PDCD4 in oral squamous cell carcinoma. Acta Biochim Biophys. Sin (Shanghai) 49, 808-816. doi: 10.1093/abbs/gmx078

Lousada-Fernandez, F., Rapado-Gonzalez, O., Lopez-Cedrun, J. L., Lopez-Lopez, R., Muinelo-Romay, L., and Suarez-Cunqueiro, M. M. (2018). Liquid biopsy in Oral Cancer. Int. J. Mol. Sci. 19:1704. doi: 10.3390/ijms19061704

Mathieu, M., Martin-Jaular, L., Lavieu, G., and Thery, C. (2019). Specificities of secretion and uptake of exosomes and other extracellular vesicles for cell-to-cell communication. Nat. Cell Biol. 21, 9-17. doi: 10.1038/s41556-018-0250-9

Mellman, I. (1996). Endocytosis and molecular sorting. Annu. Rev. Cell Dev. Biol. 12, 575-625. doi: 10.1146/annurev.cellbio.12.1.575

Miska, E. A. (2005). How microRNAs control cell division, differentiation and death. Curr. Opin. Genet. Dev. 15, 563-568. doi: 10.1016/j.gde.2005.08.005

Momen-Heravi, F., and Bala, S. (2018). Extracellular vesicles in oral squamous carcinoma carry oncogenic miRNA profile and reprogram monocytes via NF- $\kappa$ B pathway. Oncotarget 9, 34838-34854. doi: 10.18632/oncotarget.26208

Munoz, J. L., Bliss, S. A., Greco, S. J., Ramkissoon, S. H., Ligon, K. L., and Rameshwar, P. (2013). Delivery of functional anti-miR-9 by mesenchymal stem cell-derived exosomes to glioblastoma multiforme cells conferred chemosensitivity. Mol. Ther. Nucleic Acids 2:e126. doi: 10.1038/mtna.2013.60

Nahand, J. S., Mahjoubin-Tehran, M., Moghoofei, M., Pourhanifeh, M. H., Mirzaei, H. R., Asemi, Z., et al. (2020a). Exosomal miRNAs: novel players in viral infection. Epigenomics 12, 353-370. doi: 10.2217/epi-2019-0192

Nahand, J. S., Vandchali, N. R., Darabi, H., Doroudian, M., Banafshe, H. R., Moghoofei, M., et al. (2020b). Exosomal microRNAs: novel players in cervical cancer. Epigenomics 12, 1651-1660. doi: 10.2217/epi-2020-0026

Nair, S., Tang, K. D., Kenny, L., and Punyadeera, C. (2018). Salivary exosomes as potential biomarkers in cancer. Oral. Oncol. 84, 31-40. doi: 10.1016/j. oraloncology.2018.07.001

Nakase, I., and Futaki, S. (2015). Combined treatment with a pH-sensitive fusogenic peptide and cationic lipids achieves enhanced cytosolic delivery of exosomes. Sci. Rep. 5:10112. doi: 10.1038/srep10112

Nazimek, K., Bryniarski, K., and Askenase, P. W. (2016). Functions of exosomes and microbial extracellular vesicles in allergy and contact and delayed-type hypersensitivity. Int. Arch. Allergy Immunol. 171, 1-26. doi: 10.1159/00044 9249

Obuchowski, N. A., and Bullen, J. A. (2018). Receiver operating characteristic (ROC) curves: review of methods with applications in diagnostic medicine. Phys. Med. Biol. 63:07TR01. doi: 10.1088/1361-6560/aab4b1

Oeyen, E., Van Mol, K., Baggerman, G., Willems, H., Boonen, K., Rolfo, C., et al. (2018). Ultrafiltration and size exclusion chromatography combined with asymmetrical-flow field-flow fractionation for the isolation and characterisation of extracellular vesicles from urine. J. Extracell. Vesicles 7:1490143. doi: 10.1080/ 20013078.2018.1490143

Ohno, S., Ishikawa, A., and Kuroda, M. (2013a). Roles of exosomes and microvesicles in disease pathogenesis. Adv. Drug. Deliv. Rev. 65, 398-401. doi: 10.1016/j.addr.2012.07.019

Ohno, S., Takanashi, M., Sudo, K., Ueda, S., Ishikawa, A., Matsuyama, N., et al. (2013b). Systemically injected exosomes targeted to EGFR deliver antitumor microRNA to breast cancer cells. Mol. Ther. 21, 185-191. doi: 10.1038/mt.2012. 180

Ono, K., Eguchi, T., Sogawa, C., Calderwood, S. K., Futagawa, J., Kasai, T., et al. (2018). HSP-enriched properties of extracellular vesicles involve survival of metastatic oral cancer cells. J. Cell Biochem. 119, 7350-7362. doi: 10.1002/jcb. 27039
Paget, S. (1989). The distribution of secondary growths in cancer of the breast 1889. Cancer Metastasis Rev. 8, 98-101.

Pant, S., Hilton, H., and Burczynski, M. E. (2012). The multifaceted exosome: biogenesis, role in normal and aberrant cellular function, and frontiers for pharmacological and biomarker opportunities. Biochem. Pharmacol. 83, 14841494. doi: 10.1016/j.bcp.2011.12.037

Peltanova, B., Raudenska, M., and Masarik, M. (2019). Effect of tumor microenvironment on pathogenesis of the head and neck squamous cell carcinoma: a systematic review. Mol. Cancer 18:63. doi: 10.1186/s12943-0190983-5

Pourhanifeh, M. H., Mahjoubin-Tehran, M., Shafiee, A., Hajighadimi, S., Moradizarmehri, S., Mirzaei, H., et al. (2020). MicroRNAs and exosomes: small molecules with big actions in multiple myeloma pathogenesis. Iubmb Life 72, 314-333. doi: 10.1002/iub.2211

Qi, H., Liu, C., Long, L., Ren, Y., Zhang, S., Chang, X., et al. (2016). Blood exosomes endowed with magnetic and targeting properties for Cancer therapy. ACS Nano 10, 3323-3333. doi: 10.1021/acsnano.5b06939

Qiu, Y. L., Liu, Y. H., Ban, J. D., Wang, W. J., Han, M., Kong, P., et al. (2019). Pathway analysis of a genome-wide association study on a long non-coding RNA expression profile in oral squamous cell carcinoma. Oncol. Rep. 41, 895-907. doi: 10.3892/or.2018.6870

Rabinowits, G., Bowden, M., Flores, L. M., Verselis, S., Vergara, V., Jo, V. Y., et al. (2017). Comparative analysis of MicroRNA expression among benign and malignant tongue tissue and plasma of patients with Tongue Cancer. Front. Oncol. 7:191. doi: 10.3389/fonc.2017.00191

Raposo, G., and Stoorvogel, W. (2013). Extracellular vesicles: exosomes, microvesicles, and friends. J. Cell Biol. 200, 373-383. doi: 10.1083/jcb. 201211138

Reeves, M. E., Baldwin, M. L., Aragon, R., Baldwin, S., Chen, S. T., Li, X., et al. (2012). RASSF1C modulates the expression of a stem cell renewal gene. PIWIL1. BMC Res. Notes 5:239. doi: 10.1186/1756-0500-5-239

Ridder, K., Keller, S., Dams, M., Rupp, A. K., Schlaudraff, J., Del Turco, D., et al. (2014). Extracellular vesicle-mediated transfer of genetic information between the hematopoietic system and the brain in response to inflammation. PLoS Biol. 12:e1001874. doi: 10.1371/journal.pbio.1001874

Rosenberger, L., Ezquer, M., Lillo-Vera, F., Pedraza, P. L., Ortúzar, M. I., González P. L., et al. (2019). Stem cell exosomes inhibit angiogenesis and tumor growth of oral squamous cell carcinoma. Sci. Rep. 9:663. doi: 10.1038/s41598-018-36 855-6

Sadri Nahand, J., Moghoofei, M., Salmaninejad, A., Bahmanpour, Z., Karimzadeh, M., Nasiri, M., et al. (2020). Pathogenic role of exosomes and microRNAs in HPV-mediated inflammation and cervical cancer: a review. Int. J. Cancer 146, 305-320. doi: 10.1002/ijc.32688

Sakha, S., Muramatsu, T., Ueda, K., and Inazawa, J. (2016). Exosomal microRNA miR-1246 induces cell motility and invasion through the regulation of DENND2D in oral squamous cell carcinoma. Sci. Rep. 6:38750. doi: 10.1038/ srep38750

Samuel, P., Fabbri, M., and Carter, D. R. F. (2017). Mechanisms of drug resistance in cancer: the role of extracellular vesicles. Proteomics 17:1600375. doi: 10.1002/ pmic. 201600375

Shah, M. Y., and Calin, G. A. (2013). The mix of two worlds: non-coding RNAs and hormones. Nucleic Acid Ther. 23, 2-8. doi: 10.1089/nat.2012.0375

Sohn, W., Kim, J., Kang, S. H., Yang, S. R., Cho, J. Y., Cho, H. C., et al. (2015). Serum exosomal microRNAs as novel biomarkers for hepatocellular carcinoma. Exp. Mol. Med. 47:e184. doi: 10.1038/emm.2015.68

Steinbichler, T. B., Dudas, J., Skvortsov, S., Ganswindt, U., Riechelmann, H., and Skvortsova, I. I. (2019). Therapy resistance mediated by exosomes. Mol. Cancer 18:58. doi: 10.1186/s12943-019-0970-x

Stickney, Z., Losacco, J., McDevitt, S., Zhang, Z., and Lu, B. (2016). Development of exosome surface display technology in living human cells. Biochem. Biophys. Res. Commun. 472, 53-59. doi: 10.1016/j.bbrc.2016.02.058

Sun, L. P., Xu, K., Cui, J., Yuan, D. Y., Zou, B., Li, J., et al. (2019). Cancer-associated fibroblast-derived exosomal miR-382-5p promotes the migration and invasion of oral squamous cell carcinoma. Oncol. Rep. 42, 1319-1328. doi: 10.3892/or. 2019.7255

Tachibana, H., Sho, R., Takeda, Y., Zhang, X., Yoshida, Y., Narimatsu, H., et al. (2016). Circulating miR-223 in oral cancer: its potential as a novel diagnostic 
biomarker and therapeutic target. PLoS One 11:e0159693. doi: 10.1371/journal pone. 0159693

Tahiri, A., Leivonen, S. K., Lüders, T., Steinfeld, I., Ragle Aure, M., Geisler, J., et al. (2014). Deregulation of cancer-related miRNAs is a common event in both benign and malignant human breast tumors. Carcinogenesis 35, 76-85. doi: $10.1093 /$ carcin/bgt333

Thery, C., Witwer, K. W., Aikawa, E., Alcaraz, M. J., Anderson, J. D., Andriantsitohaina, R., et al. (2018). Minimal information for studies of extracellular vesicles 2018 (MISEV2018): a position statement of the international society for extracellular vesicles and update of the MISEV2014 guidelines. J. Extracell Vesicles 7:1535750. doi: 10.1080/20013078.2018.1535750

Tschuschke, M., Kocherova, I., Bryja, A., Mozdziak, P., Angelova Volponi, A., Janowicz, K., et al. (2020). Inclusion biogenesis, methods of isolation and clinical application of human cellular exosomes. J. Clin. Med. 9:436. doi: 10. $3390 / \mathrm{jcm} 9020436$

Villarroya-Beltri, C., Gutiérrez-Vázquez, C., Sánchez-Cabo, F., Pérez-Hernández, D., Vázquez, J., Martin-Cofreces, N., et al. (2013). Sumoylated hnRNPA2B1 controls the sorting of miRNAs into exosomes through binding to specific motifs. Nat. Commun. 4:2980. doi: 10.1038/ncomms3980

Vu, L. T., Peng, B., Zhang, D. X., Ma, V., Mathey-Andrews, C. A., Lam, C. K., et al. (2019). Tumor-secreted extracellular vesicles promote the activation of cancerassociated fibroblasts via the transfer of microRNA-125b. J. Extracell Vesicles 8:1599680. doi: 10.1080/20013078.2019.1599680

Vychytilova-Faltejskova, P., Stitkovcova, K., Radova, L., Sachlova, M., Kosarova, Z., Slaba, K., et al. (2018). Circulating PIWI-Interacting RNAs piR-5937 and piR28876 are promising diagnostic biomarkers of Colon Cancer. Cancer Epidemiol. Biomarkers Prev. 27, 1019-1028. doi: 10.1158/1055-9965.EPI-18-0318

Wang, A., Liu, J., Zhuang, X., Yu, S., Zhu, S., Liu, Y., et al. (2020). Identification and comparison of piRNA expression profiles of exosomes derived from human stem cells from the apical papilla and bone marrow mesenchymal stem cells. Stem Cells Dev. 29, 511-520. doi: 10.1089/scd.2019.0277

Wang, H., Hou, L., Li, A., Duan, Y., Gao, H., and Song, X. (2014). Expression of serum exosomal microRNA-21 in human hepatocellular carcinoma. Biomed Res. Int. 2014:864894. doi: 10.1155/2014/864894

Wang, J., Lv, B., Su, Y., Wang, X., Bu, J., and Yao, L. (2019a). Exosome-Mediated transfer of IncRNA HOTTIP promotes cisplatin resistance in gastric cancer cells by regulating HMGA1/miR-218 axis. Onco Targets Ther. 12, 11325-11338. doi: 10.2147/OTT.S231846

Wang, X., Qin, X., Yan, M., Shi, J., Xu, Q., Li, Z., et al. (2019b). Loss of exosomal miR-3188 in cancer-associated fibroblasts contributes to HNC progression. J. Exp. Clin. Cancer Res. 38:151. doi: 10.1186/s13046-019-1144-9

Wang, J., Zheng, Y., and Zhao, M. (2016). Exosome-Based cancer therapy: implication for targeting Cancer stem cells. Front. Pharmacol. 7:533. doi: 10. 3389/fphar.2016.00533

Wang, Y., Sun, C., Li, T., Xu, H., Zhou, Y., Dan, H., et al. (2014). Integrative approach detected association between genetic variants of microRNA binding sites of TLRs pathway genes and OSCC susceptibility in Chinese Han population. PLoS One 9:e101695. doi: 10.1371/journal.pone. 0101695

Weber, J. A., Baxter, D. H., Zhang, S., Huang, D. Y., Huang, K. H., Lee, M. J., et al. (2010). The microRNA spectrum in 12 body fluids. Clin. Chem. 56, 1733-1741. doi: 10.1373/clinchem.2010.147405

Weick, E. M., and Miska, E. A. (2014). piRNAs: from biogenesis to function. Development 141, 3458-3471. doi: 10.1242/dev.094037

Wu, M., Ouyang, Y., Wang, Z., Zhang, R., Huang, P. H., Chen, C., et al. (2017). Isolation of exosomes from whole blood by integrating acoustics and microfluidics. Proc. Natl. Acad. Sci. U S A. 114, 10584-10589. doi: 10.1073/pnas. 1709210114

Wu, S., Zheng, Q., Xing, X., Dong, Y., Wang, Y., You, Y., et al. (2018). Matrix stiffness-upregulated LOXL2 promotes fibronectin production, MMP9 and CXCL12 expression and BMDCs recruitment to assist pre-metastatic niche formation. J. Exp. Clin. Cancer Res. 37:99. doi: 10.1186/s13046-0180761-z

Xie, C., Du, L. Y., Guo, F., Li, X., and Cheng, B. (2019a). Exosomes derived from microRNA-101-3p-overexpressing human bone marrow mesenchymal stem cells suppress oral cancer cell proliferation, invasion, and migration. Mol. Cell Biochem. 458, 11-26. doi: 10.1007/s11010-019-03526-7

Xie, C., Ji, N., Tang, Z., Li, J., and Chen, Q. (2019b). The role of extracellular vesicles from different origin in the microenvironment of head and neck cancers. Mol. Cancer 18:83. doi: 10.1186/s12943-019-0985-3

Xie, X., Guo, P., Yu, H., Wang, Y., and Chen, G. (2018). Ribosomal proteins: insight into molecular roles and functions in hepatocellular carcinoma. Oncogene 37, 277-285. doi: 10.1038/onc.2017.343

Xu, H., Guo, S., Li, W., and Yu, P. (2015). The circular RNA Cdrlas, via miR-7 and its targets, regulates insulin transcription and secretion in islet cells. Sci. Rep. 5:12453. doi: 10.1038/srep12453

Xu, H., Yang, Y., Zhao, H., Yang, X., Luo, Y., Ren, Y., et al. (2016). Serum miR483-5p: a novel diagnostic and prognostic biomarker for patients with oral squamous cell carcinoma. Tumour. Biol. 37, 447-453. doi: 10.1007/s13277-0153514-z

Xu, Y., Shen, L. F., Li, F. J., Yang, J. W., Wan, X. P., and Ouyang, M. (2019). microRNA-16-5p-containing exosomes derived from bone marrow-derived mesenchymal stem cells inhibit proliferation, migration, and invasion, while promoting apoptosis of colorectal cancer cells by downregulating ITGA2. J. Cell. Physiol. 234, 21380-21394. doi: 10.1002/jcp.28747

Xue, V. W., Wong, C. S. C., and Cho, W. C. S. (2019). Early detection and monitoring of cancer in liquid biopsy: advances and challenges. Expert Rev. Mol. Diagn. 19, 273-276. doi: 10.1080/14737159.2019.1583104

Ye, S. B., Li, Z. L., Luo, D. H., Huang, B. J., Chen, Y. S., Zhang, X. S., et al. (2014). Tumor-derived exosomes promote tumor progression and T-cell dysfunction through the regulation of enriched exosomal microRNAs in human nasopharyngeal carcinoma. Oncotarget 5, 5439-5452. doi: 10.18632/oncotarget. 2118

Yuan, L., Liu, Y. Q., Qu, Y. H., Liu, L., and Li, H. X. (2019). Exosomes derived from microrna-148b-3p-overexpressing human umbilical cord mesenchymal stem cells restrain Breast Cancer progression. Front. Oncol. 9:1076. doi: 10.3389/fonc. 2019.01076

Yuan, T., Huang, X., Woodcock, M., Du, M., Dittmar, R., Wang, Y., et al. (2016). Plasma extracellular RNA profiles in healthy and cancer patients. Sci. Rep. 6:19413. doi: 10.1038/srep19413

Zhang, H., Deng, T., Ge, S., Liu, Y., Bai, M., Zhu, K., et al. (2019). Exosome circRNA secreted from adipocytes promotes the growth of hepatocellular carcinoma by targeting deubiquitination-related USP7. Oncogene 38, 2844-2859. doi: 10. 1038/s41388-018-0619-Z

Zhang, J., Li, S., Li, L., Li, M., Guo, C., Yao, J., et al. (2015). Exosome and exosomal microRNA: trafficking, sorting, and function. Genom. Proteom. Bioinform. 13, 17-24. doi: 10.1016/j.gpb.2015.02.001

Zhang, Y., Bi, J., Huang, J., Tang, Y., Du, S., and Li, P. (2020). Exosome: a review of its classification, isolation techniques, storage, diagnostic and targeted therapy applications. Int. J. Nanomed. 15, 6917-6934. doi: 10.2147/IJN.S264498

Zhao, M., Xu, J., Zhong, S., Liu, Y., Xiao, H., Geng, L., et al. (2019). Expression profiles and potential functions of circular RNAs in extracellular vesicles isolated from radioresistant glioma cells. Oncol. Rep. 41, 1893-1900. doi: 10. 3892/or.2019.6972

Zlotogorski-Hurvitz, A., Dayan, D., Chaushu, G., Salo, T., and Vered, M. (2016). Morphological and molecular features of oral fluid-derived exosomes: oral cancer patients versus healthy individuals. J. Cancer Res. Clin. Oncol. 142, 101-110. doi: 10.1007/s00432-015-2005-3

Conflict of Interest: The authors declare that the research was conducted in the absence of any commercial or financial relationships that could be construed as a potential conflict of interest.

Copyright $\odot 2021$ Lu, Zheng, Yuan, Pathak, Yang, Wang, Ye, Cho, Zeng and Wu. This is an open-access article distributed under the terms of the Creative Commons Attribution License (CC BY). The use, distribution or reproduction in other forums is permitted, provided the original author(s) and the copyright owner(s) are credited and that the original publication in this journal is cited, in accordance with accepted academic practice. No use, distribution or reproduction is permitted which does not comply with these terms. 